\title{
REMARKS ON A WIENER TYPE PSEUDODIFFERENTIAL ALGEBRA AND FOURIER INTEGRAL OPERATORS
}

\section{A. BOUlKhemair}

\section{Introduction}

In [1], Sjöstrand introduced a general class of symbols containing Hörmander's class $S_{0,0}^{0}$, without any reference to derivatives and allowing composition and $L^{2}$ boundedness for the associated pseudodifferential operators. In [2], he denoted it by $S_{w}$ and, among other things, proved that $\operatorname{Op}\left(S_{w}\right)$ is a Wiener algebra: If $A \in \mathrm{Op}\left(S_{w}\right)$ is invertible in $\mathcal{L}\left(L^{2}\right)$, then $A^{-1} \in \mathrm{Op}\left(S_{w}\right)$. Let us recall that a function $u: \mathbb{R}^{n} \rightarrow \mathbb{C}$ is in $S_{w}\left(\mathbb{R}^{n}\right)$ if, for some $\chi \in \mathcal{S}\left(\mathbb{R}^{n}\right)$ with non zero integral,

$$
\xi \mapsto \sup _{k \in \mathbb{R}^{n}}\left|\mathcal{F}\left(u \tau_{k \chi}\right)(\xi)\right|
$$

is an integrable function in $\mathbb{R}^{n}$. Here, $\mathcal{F}$ denotes the Fourier transformation and $\tau_{k} \chi(x)=\chi(x-k)$. Provided with the norm equal to the Lebesgue integral of $(0.1), S_{w}$ is a Banach space. Notice that changing $\chi$ gives rise to an equivalent norm.

In [3], we studied $L^{2}$ estimates for various classes of symbols of limited regularity. Among these classes, we defined a class which we denoted by $\mathcal{A}$ and proved that $\mathcal{A}$ is an algebra which allows $L^{2}$ boundedness for the standard quantization. Recall that a function $u: \mathbb{R}^{n} \rightarrow \mathbb{C}$ is in $\mathcal{A}\left(\mathbb{R}^{n}\right)$ if, for some $\chi \in \mathcal{S}\left(\mathbb{R}^{n}\right)$ with non zero integral,

$$
k \mapsto \sup _{x \in \mathbb{R}^{n}}\left|\mathcal{F}^{-1}\left[\mathcal{F}(u) \tau_{k} \chi\right](x)\right|
$$

is an integrable function in $\mathbb{R}^{n}$. Here again, the Lebesgue integral of (0.2) defines a norm on $\mathcal{A}$ that makes it complete and another choice for $\chi$ gives rise to an equivalent norm.

In this paper, we first remark that the classes $S_{w}$ and $\mathcal{A}$ coincide. The main consequence of this is a theorem of structure, Theorem 1.2 below or its corollary, which improves our understanding of $S_{w}$ and gives a new point of view for it, a spectral one. In particular, it shows that each element of $S_{w}$ can be written, in a natural manner, as a uniformly convergent series (or integral) of symbols

Received June 12, 1996. 
in $S_{0,0}^{0}$. This is the subject of $\S 1$. We show afterwards that the spectral point of view can be useful in the pseudodifferential analysis of the symbol class $S_{w}$. (See $\S 2$ and $\S 4$ ).

The second remark in this work is that one can define well-behaving Fourier integral operators using the $S_{w}$ regularity. For example, if we take amplitudes and phases with that regularity, we obtain Fourier integral operators which are $L^{2}$ continuous and which allow composition at least formally. We also consider here the case of Fourier integrals with a large parameter in the phase, a case of interest in the applications. (See $\S 3$ ).

We end this paper with a last and short remark on $S_{w}$ which may be considered as a "negative" one. This is done in $\S 5$ and we explain there why such a remarkable class is, however, not invariant in general under the action of a change of variables.

\section{Some notations.}

- If $\alpha, \beta, \ldots$, are multi-indices, we sometimes write $\partial_{1}^{\alpha} a, \partial_{1}^{\alpha} \partial_{2}^{\beta} a, \ldots$, instead of $\partial_{x}^{\alpha} a(x, y, \ldots), \partial_{x}^{\alpha} \partial_{y}^{\beta} a(x, y, \ldots), \ldots$

- "cst" denotes a positive constant that may change from one inequality to the other.

- $|| s,. s \in R$, denotes the norm in the Sobolev space $H^{s}$.

- $\mathcal{L}\left(L^{2}\right)$ is the space of bounded operators in $L^{2}$.

- If $x \in \mathbb{R}^{n},\langle x\rangle=\sqrt{1+x^{2}}$.

\section{A spectral point of view}

Our first remark is the following :

Theorem 1.1. The classes $S_{w}$ and $\mathcal{A}$ coincide.

Proof. Let $\chi$ be in $\mathcal{S}\left(\mathbb{R}^{n}\right)$. For $u \in \mathcal{S}^{\prime}\left(\mathbb{R}^{n}\right)$, we can write, in a distributional sense,

$$
\chi(D-k) u(x)=(2 \pi)^{-n} \int \mathrm{e}^{i x \xi} \chi(\xi-k) \widehat{u}(\xi) d \xi .
$$

The Fourier transform of $\xi \mapsto \mathrm{e}^{i x \xi} \chi(\xi-k)$ is equal to $y \mapsto \mathrm{e}^{i(x-y) k} \widehat{\chi}(y-x)$, so that

$$
\chi(D-k) u(x)=(2 \pi)^{-n} \int \mathrm{e}^{i(x-y) k} \widehat{\chi}(y-x) u(y) d y=\mathrm{e}^{i x k}(2 \pi)^{-n} \mathcal{F}\left(u \tau_{x} \widehat{\chi}\right)(k) .
$$

Hence, if we choose $\chi(\xi)=\mathrm{e}^{-\frac{\xi^{2}}{2}}$ so that $\widehat{\chi}=(2 \pi)^{\frac{n}{2}} \chi$, we obtain

$$
\sup _{x \in \mathbb{R}^{n}}|\chi(D-k) u(x)|=(2 \pi)^{-\frac{n}{2}} \sup _{x \in \mathbb{R}^{n}}\left|\mathcal{F}\left(u \tau_{x} \chi\right)(k)\right|,
$$

and the theorem follows since the spaces $\mathcal{A}$ and $S_{w}$ do not depend on the choice of the test function $\chi$.

This result allows us to give a spectral characterization of $S_{w}$ that is similar to the well known characterizations of functional spaces by means of dyadic techniques, the annuli being replaced here by "cubes". 
Theorem 1.2. If $u \in \mathcal{S}^{\prime}\left(\mathbb{R}^{n}\right)$, then, $u \in S_{w}\left(\mathbb{R}^{n}\right)$ if and only if there exists a compact set $Q \subset \mathbb{R}^{n}$ and a sequence $\left(u_{k}\right)_{k \in \mathbb{Z}^{n}}$ in $L^{\infty}\left(\mathbb{R}^{n}\right)$ such that $\operatorname{supp}\left(\widehat{u}_{k}\right) \subset$ $k+Q, \sum_{k}\left\|u_{k}\right\|_{L^{\infty}}<\infty$ and $u=\sum_{k} u_{k}$.

Proof. Necessity : If $u \in S_{w}\left(\mathbb{R}^{n}\right)$, take $u_{k}=\chi(D-k) u$ with $\chi \in \mathcal{D}\left(\mathbb{R}^{n}\right)$ and $\sum_{k} \tau_{k} \chi=1$. In fact, if $\chi_{1} \in \mathcal{D}\left(\mathbb{R}^{n}\right)$ and $\int \chi_{1}(\xi) d \xi=1$, one can write, with some appropriate $\psi \in \mathcal{D}\left(\mathbb{R}^{n}\right)$,

$$
\chi(D-k) u(x)=\int \psi(k-l) \chi(D-k) \chi_{1}(D-l) u(x) d l, \quad x \in \mathbb{R}^{n} .
$$

Hence,

$$
\begin{aligned}
\sum_{k}\|\chi(D-k) u\|_{L^{\infty}} & \leq \sup _{l} \sum_{k}|\psi(k-l)|\left\|\mathcal{F}^{-1}(\chi)\right\|_{L^{1}} \int\left\|\chi_{1}(D-l) u\right\|_{L^{\infty}} d l \\
& \leq \operatorname{cst}\|u\|_{S_{w}} .
\end{aligned}
$$

Sufficiency : Take $\chi \in \mathcal{D}\left(\mathbb{R}^{n}\right)$. It follows from the hypothesis on $\operatorname{supp}\left(u_{k}\right)$ that there exists $\psi \in \mathcal{D}\left(\mathbb{R}^{n}\right)$ such that

$$
\chi(D-l) u=\sum_{k} \psi(k-l) \chi(D-l) u_{k},
$$

so that,

$$
\int\|\chi(D-l) u\|_{L^{\infty}} d l \leq \sum_{k} \int|\psi(k-l)|\left\|\mathcal{F}^{-1}(\chi)\right\|_{L^{1}}\left\|u_{k}\right\|_{L^{\infty}} d l=\operatorname{cst} \sum_{k}\left\|u_{k}\right\|_{L^{\infty}} .
$$

Thus, the theorem is proved.

Corollary 1.3. A tempered distribution $u$ is in $S_{w}\left(\mathbb{R}^{n}\right)$ if and only if there exists a compact set $Q \subset \mathbb{R}^{n}$ and a sequence $\left(u_{k}\right)_{k \in \mathbb{Z}^{n}}$ in $L^{\infty}\left(\mathbb{R}^{n}\right)$ such that $\operatorname{supp}\left(\widehat{u}_{k}\right) \subset Q, \sum_{k}\left\|u_{k}\right\|_{L^{\infty}}<\infty$ and $u(x)=\sum_{k} \mathrm{e}^{i x k} u_{k}(x)$.

Notice here the analogy with absolutely convergent Fourier series. In fact, one can show that each element of $S_{w}$ can be written locally as the sum of a true absolutely convergent Fourier series. What is interesting in this corollary is that, the sequence $\left(u_{k}\right)_{k \in \mathbb{Z}^{n}}$ is bounded in $S_{0,0}^{0}$ and this will be exploited in the sequel. One can replace the series in Theorem 1.2 and Corollary 1.3 by (Lebesgue) integrals and the sequences $\left(u_{k}\right)_{k \in \mathbb{Z}^{n}}$ by families $\left(u_{k}\right)_{k \in \mathbb{R}^{n}}$. We do not give details since they are not needed.

\section{On $L^{2}$ boundedness}

As a first example of application of the above spectral characterization, we give a simple and direct proof of the $L^{2}$ continuity of the operator $\mathrm{Op}_{t}(a)$ defined by

$$
\left[O p_{t}(a) v\right](x)=(2 \pi)^{-n} \int_{\mathbb{R}^{2 n}} \mathrm{e}^{i(x-y) \eta} a(t x+(1-t) y, \eta) v(y) d y d \eta, \quad v \in \mathcal{S}\left(\mathbb{R}^{n}\right),
$$


where $0 \leq t \leq 1$ and $a \in S_{w}\left(\mathbb{R}^{2 n}\right)$.

The idea of proof is that of [3] where the case $t=1$ is discussed. The integral above has a meaning in $\mathcal{S}^{\prime}$ and, for example, one can define it by introducing a factor $\psi(\epsilon \eta), \psi \in \mathcal{S}\left(\mathbb{R}^{n}\right), \psi(0)=1$, and by taking the limit when $\epsilon \rightarrow 0$.

Set $I=\left\langle\mathrm{Op}_{t}(a) v, u\right\rangle_{\mathcal{S}^{\prime}, \mathcal{S}}, u \in \mathcal{S}\left(\mathbb{R}^{n}\right)$. It follows from Corollary 1.3 that one can write

$$
a(x, \eta)=\sum_{k, l \in \mathbb{Z}^{n}} \mathrm{e}^{i(k x+l \eta)} a_{k l}(x, \eta)
$$

where $a_{k l}$ is bounded in $S_{0,0}^{0}$ and $\sum_{k, l}\left\|a_{k l}\right\|_{L^{\infty}}<\infty$. A simple computation gives $I=\sum_{k, l} I_{k l}$ with $I_{k l}=\left\langle\mathrm{Op}_{t}\left(a_{k l}\right) v_{k l}, u_{k l}\right\rangle, u_{k l}(x)=u(x-(1-t) l) \mathrm{e}^{i t x k}$ and $v_{k l}(y)=v(y+t l) \mathrm{e}^{i(1-t) y k}$. Now, apply the well known $L^{2}$ estimates for $S_{0,0}^{0}$ type symbols :

$$
\left|I_{k l}\right| \leq \operatorname{cst} \sum_{|\alpha|,|\beta| \leq N}\left\|\partial_{x}^{\alpha} \partial_{\eta}^{\beta} a_{k l}\right\|_{L^{\infty}}\left\|u_{k l}\right\|_{L^{2}}\left\|v_{k l}\right\|_{L^{2}}
$$

with $N$ a sufficiently large integer. Finally, since the spectrum of $a_{k l}$ is contained in a fixed compact set, we have $\left\|\partial_{x}^{\alpha} \partial_{\eta}^{\beta} a_{k l}\right\|_{L^{\infty}} \leq$ cst $\left\|a_{k l}\right\|_{L^{\infty}}$, so that,

$$
|I| \leq \sum_{k, l}\left|I_{k l}\right| \leq \operatorname{cst} \sum_{k, l}\left\|a_{k l}\right\|_{L^{\infty}}\|u\|_{L^{2}}\|v\|_{L^{2}} \leq \operatorname{cst}\|a\|_{S_{w}}\|u\|_{L^{2}}\|v\|_{L^{2}} .
$$

The same argument works for the more general operator

$$
A v(x)=(2 \pi)^{-n} \int_{\mathbb{R}^{2 n}} \mathrm{e}^{i(x-y) \eta} a(x, y, \eta) v(y) d y d \eta, \quad v \in \mathcal{S}\left(\mathbb{R}^{n}\right),
$$

when $a \in S_{w}\left(\mathbb{R}^{3 n}\right)$. In fact, the above method of proof can be used to handle even more general oscillating integrals. Indeed, consider an integral of the form

$$
A v(x)=\int_{\mathbb{R}^{n+\nu}} \mathrm{e}^{i \varphi(x, y, \theta)} a(x, y, \theta) v(y) d y d \theta,
$$

where $v \in \mathcal{S}\left(\mathbb{R}^{n}\right), a \in S_{w}\left(\mathbb{R}^{n} \times \mathbb{R}^{n} \times \mathbb{R}^{\nu}\right)$ and $\varphi: \mathbb{R}^{n} \times \mathbb{R}^{n} \times \mathbb{R}^{\nu} \rightarrow \mathbb{R}$ satisfies : $\partial^{\alpha} \varphi \in S_{0,0}^{0}$ for all multi-indices $\alpha$ such that $|\alpha|=2$, and

$$
\left|\operatorname{det}\left(\begin{array}{cc}
\partial_{x} \partial_{y} \varphi & \partial_{\theta} \partial_{y} \varphi \\
\partial_{x} \partial_{\theta} \varphi & \partial_{\theta}^{2} \varphi
\end{array}\right)\right| \geq \delta
$$

with some constant $\delta>0$. Notice that this last condition implies that the maps

$$
(x, \theta) \mapsto\left(\partial_{y} \varphi(x, y, \theta), \partial_{\theta} \varphi(x, y, \theta)\right) \text { and }(y, \theta) \mapsto\left(\partial_{x} \varphi(x, y, \theta), \partial_{\theta} \varphi(x, y, \theta)\right)
$$

are global diffeomorphisms from $\mathbb{R}^{n} \times \mathbb{R}^{\nu}$ onto itself.

This type of integrals was studied by Asada and Fujiwara in [4] when $a \in S_{0,0}^{0}$. 
If the decomposition

$$
a(x, y, \theta)=\sum_{(k, l, m) \in \mathbb{Z}^{2 n+\nu}} \mathrm{e}^{i(k x+l y+m \theta)} a_{k l m}(x, y, \theta)
$$

is that given by Corollary 1.3, we can define the operator $A$ by

$$
A v(x)=\sum_{(k, l, m) \in \mathbb{Z}^{2 n+\nu}} \mathrm{e}^{i k x} \int_{\mathbb{R}^{n+\nu}} \mathrm{e}^{i(\varphi(x, y, \theta)+m \theta)} a_{k l m}(x, y, \theta) \mathrm{e}^{i l y} v(y) d y d \theta,
$$

the integrals being classical oscillating integrals (since $a_{k l m} \in S_{0,0}^{0}$ ) and the convergence of the series being checked in $\mathcal{S}^{\prime}$. One can show afterwards that this definition agrees with the more classical one which consists in introducing a factor $\psi(\epsilon \theta), \psi \in \mathcal{S}\left(\mathbb{R}^{\nu}\right), \psi(0)=1$, in the integral and then taking the limit (in $\mathcal{S}^{\prime}$ ) when $\epsilon \rightarrow 0$.

Now, since the phase $\varphi(x, y, \theta)+m \theta$ satisfies the same conditions as $\varphi$, applying the result of [4] on $L^{2}$ boundedness, we obtain

$$
|<A v, u>| \leq \operatorname{cst} \sum_{(k, l, m) \in \mathbb{Z}^{2 n+\nu}} \sup _{|\alpha| \leq N}\left\|\partial^{\alpha} a_{k l m}\right\|_{L^{\infty}}\|u\|_{L^{2}}\|v\|_{L^{2}},
$$

with a sufficiently large integer $N$, and since the spectrum of $a_{k l m}$ is contained in a fixed compact set, we conclude as in the preceding proof and obtain an $L^{2}$ estimate for the global Fourier integral operator (2.2).

Moreover, applying the same argument and the result of [5] instead of that of [4], one may relax the regularity condition on the phase $\varphi$ and require, for example, only that $\partial^{\alpha} \varphi$ is in the (global) Hölder space $C^{n+\nu+\varrho}\left(\mathbb{R}^{2 n+\nu}\right), \varrho>0$, for all multi-indices $\alpha$ such that $|\alpha|=2$.

We state now the most general result we can prove on $L^{2}$ boundedness of Fourier integral operators by means of the spectral characterization.

Theorem 2.1. Assume that

(i) $a \in S_{w}\left(\mathbb{R}^{2 n+\nu}\right)$

(ii) $\partial^{\alpha} \varphi \in H_{u l}^{s, s^{\prime}, \sigma}\left(\mathbb{R}^{n} \times \mathbb{R}^{n} \times \mathbb{R}^{\nu}\right), s, s^{\prime}>\frac{n}{2}, \sigma>\nu,|\alpha|=2$.

(iii) $\varphi$ satisfies the non-degeneracy condition (2.3).

Let $A$ be given by (2.2) with the meaning (2.4). Then, $A$ defines a bounded operator in $L^{2}\left(\mathbb{R}^{n}\right)$ and

$$
\|A\|_{\mathcal{L}\left(L^{2}\right)} \leq \frac{\mathrm{cst}}{\delta} \exp \left(\operatorname{cst}\left|\varphi^{\prime \prime}\right|_{s, \sigma, s^{\prime} ; u l}\right)\|a\|_{S_{w}}
$$

the constant being independent of $(a, \varphi, \delta)$.

Recall that $H^{s, s^{\prime}, \sigma}\left(\mathbb{R}^{n} \times \mathbb{R}^{n} \times \mathbb{R}^{\nu}\right)$ is the space of tempered distributions $a(x, y, \theta)$ for which the integral

$$
|a|_{s, s^{\prime}, \sigma}^{2}=\int\left|\left(1-\Delta_{x}\right)^{\frac{s}{2}}\left(1-\Delta_{y}\right)^{\frac{s^{\prime}}{2}}\left(1-\Delta_{\theta}\right)^{\frac{\sigma}{2}}[a(x, y, \theta)]\right|^{2} d x d y d \theta
$$


is finite, and that $H_{u l}^{s, s^{\prime}, \sigma}\left(\mathbb{R}^{n} \times \mathbb{R}^{n} \times \mathbb{R}^{\nu}\right)$ is the space of tempered distributions $a(x, y, \theta)$ that are locally in $H^{s, s^{\prime}, \sigma}\left(\mathbb{R}^{n} \times \mathbb{R}^{n} \times \mathbb{R}^{\nu}\right)$ and such that the quantity

$$
|a|_{s, s^{\prime}, \sigma ; u l}=\sup _{z \in \mathbb{R}^{2 n+\nu}}\left|a \tau_{z} \chi\right|_{s, s^{\prime}, \sigma}
$$

is finite for some fixed $\chi \in \mathcal{D}\left(\mathbb{R}^{2 n+\nu}\right)$ with non zero integral. See [5] for more details. Condition (ii) in the statement above is satisfied if, for instance, $\varphi^{\prime \prime} \in H_{u l}^{n+\nu+\varrho}\left(\mathbb{R}^{2 n+\nu}\right), \varrho>0$, (uniformly local Sobolev space), or if $\varphi^{\prime \prime} \in$ $C^{n+\nu+\varrho}\left(\mathbb{R}^{2 n+\nu}\right), \varrho>0$, (global Hölder space).

Proof of Theorem 2.1. We have just to take again the argument developed above and to apply to the integrals in (2.4) Theorem 2 of [5] instead of the $L^{2}$ boundedness result of [4].

\section{More on Fourier integral operators}

In this section, we continue to give precise $L^{2}$ estimates for global non degenerate Fourier integral operators related to the class $S_{w}$. The results presented here are not applications of the spectral characterization of $S_{w}$ as is Theorem 2.1, and need proofs that are more evolved than that of Theorem 2.1. This is the reason for stating them in an independent section.

Theorem 3.1. Let $a$ be in $S_{w}\left(\mathbb{R}^{2 n+\nu}\right)$ and let $\varphi: \mathbb{R}^{2 n+\nu} \rightarrow \mathbb{R}$ be such that $\partial^{\alpha} \varphi \in S_{w}\left(\mathbb{R}^{2 n+\nu}\right)$ for $|\alpha|=2$. Assume also that $\varphi$ satisfies the non-degeneracy condition (2.3) and define the operator $A$ by

$$
A v(x)=\lim _{\epsilon \rightarrow 0} \int_{\mathbb{R}^{n+\nu}} \mathrm{e}^{i \varphi(x, y, \theta)} a(x, y, \theta) \psi(\epsilon \theta) v(y) d y d \theta, \quad\left(\text { limit in } \mathcal{S}^{\prime}\left(\mathbb{R}^{n}\right)\right),
$$

$v \in \mathcal{S}\left(\mathbb{R}^{n}\right), \psi \in \mathcal{S}\left(\mathbb{R}^{\nu}\right), \psi(0)=1,0<\epsilon \leq 1$

Then, $A$ is an operator from $\mathcal{S}\left(\mathbb{R}^{n}\right)$ to $\mathcal{S}^{\prime}\left(\mathbb{R}^{n}\right)$ which does not depend on $\psi$ and which extends uniquely to a bounded operator in $L^{2}\left(\mathbb{R}^{n}\right)$. Moreover, one can estimate the operator norm as follows,

$$
\|A\|_{\mathcal{L}\left(L^{2}\right)} \leq \frac{\mathrm{cst}}{\delta} \exp \left(\operatorname{cst}\left\|\varphi^{\prime \prime}\right\|_{S_{w}}\right)\|a\|_{S_{w}}
$$

the constant being independent of $(a, \varphi, \delta)$.

Proof. Unfortunately, we have not been able to produce a proof as simple as that of Theorem 2.1. We follow here the method of proof of Theorem 2 of [5] but not its scheme. Set $a_{\epsilon}(x, y, \theta)=a(x, y, \theta) \psi(\epsilon \theta)$ and

$$
I_{\epsilon}=\int_{\mathbb{R}^{2 n+\nu}} \mathrm{e}^{i \varphi(x, y, \theta)} a_{\epsilon}(x, y, \theta) u(x) v(y) d x d y d \theta \text { where } u, v \in \mathcal{S}\left(\mathbb{R}^{n}\right) .
$$


We can write

$$
I_{\epsilon}=\int \mathrm{e}^{i \varphi(x+k, y+l, \theta+m)} a_{\epsilon k l m}(x, y, \theta) u_{k}(x) v_{l}(y) d x d y d \theta d k d l d m
$$

with $f, g \in \mathcal{D}\left(\mathbb{R}^{n}\right), h \in \mathcal{D}\left(\mathbb{R}^{\nu}\right), \int f=\int g=\int h=1, u_{k}(x)=\tilde{f}(x) u(x+k)$, $\tilde{f} \in \mathcal{D}\left(\mathbb{R}^{n}\right), \quad \tilde{f}=1$ on $\operatorname{supp}(f), v_{l}(y)=\widetilde{g}(y) v(y+l), \widetilde{g} \in \mathcal{D}\left(\mathbb{R}^{n}\right), \quad \widetilde{g}=1$ on $\operatorname{supp}(g)$, and $a_{\epsilon k l m}(x, \theta, y)=f(x) g(y) h(\theta) a_{\epsilon}(x+k, y+l, \theta+m)$.

Set $z=(x, y, \theta), q=(k, l, m)$ and write the following Taylor formula :

$$
\varphi(z+q)=\varphi(q)+\partial_{1} \varphi(q) x+\partial_{2} \varphi(q) y+\partial_{3} \varphi(q) \theta+\varphi_{q}(z)
$$

with $\varphi_{q}(z)=\int_{0}^{1}(1-t) \varphi^{\prime \prime}(q+t z) z^{2} d t$. To study this remainder, we need the following property of $S_{w}$. This will also be needed later on.

Proposition 3.2. Set $u_{t}(x)=u(t x)$. If $u \in S_{w}\left(\mathbb{R}^{n}\right)$, then, $u_{t} \in S_{w}\left(\mathbb{R}^{n}\right)$, and there exists a constant $C>0$ such that, for all $u \in S_{w}\left(\mathbb{R}^{n}\right)$ and $t \in[0,1]$, $\left\|u_{t}\right\|_{S_{w}} \leq C\|u\|_{S_{w}}$.

Proof. If $\chi \in \mathcal{D}\left(\mathbb{R}^{n}\right)$ and $\int \chi(\xi) d \xi=1$, we can write

$$
\begin{aligned}
\chi(D-k) u_{t}(x) & =(2 \pi)^{-n} \int \mathrm{e}^{i t x \xi} \chi(t \xi-k) \chi(\xi-l) \widehat{u}(\xi) d \xi d l \\
& =\int \tilde{\chi}(k-t l)[\chi(t D-k) \chi(D-l) u](t x) d l
\end{aligned}
$$

with some appropriate $\tilde{\chi} \in \mathcal{D}\left(\mathbb{R}^{n}\right)$ since $k-t l=t(\xi-l)-(t \xi-k)$ lies in some fixed compact set. Hence,

$$
\begin{aligned}
\int\left\|\chi(D-k) u_{t}\right\|_{L^{\infty}} d k & \leq \int|\widetilde{\chi}(k-t l)|\left\|\mathcal{F}^{-1}(\chi)\right\|_{L^{1}}\|\chi(D-l) u\|_{L^{\infty}} d l d k \\
& \leq\|\widetilde{\chi}\|_{L^{1}}\left\|\mathcal{F}^{-1}(\chi)\right\|_{L^{1}} \int\|\chi(D-l) u\|_{L^{\infty}} d l
\end{aligned}
$$

and this establishes the proposition.

Since we can write $\varphi_{q}(z)=\int_{0}^{1}(1-t)\left(\varphi^{\prime \prime}\right)_{t}\left(\frac{q}{t}+z\right) z^{2} d t$, it follows from Proposition 3.2 that, for all $\chi \in \mathcal{D}\left(\mathbb{R}^{2 n+\nu}\right)$,

$$
\int \sup _{q}\left|\widehat{\chi \varphi_{q}}(\zeta)\right| d \zeta \leq \operatorname{cst}\left\|\varphi^{\prime \prime}\right\|_{S_{w}}
$$

Moreover, if we set $b_{\epsilon, q}=a_{\epsilon, q} \mathrm{e}^{i \varphi_{q}}$, one can check easily that

$$
\int \sup _{q}\left|\widehat{b_{\epsilon, q}}(\zeta)\right| d \zeta \leq \operatorname{cst}\left\|a_{\epsilon}\right\|_{S_{w}} \exp \left(\operatorname{cst}\left\|\varphi^{\prime \prime}\right\|_{S_{w}}\right), \quad 0 \leq \epsilon \leq 1
$$


Now, introducing (3.2) into (3.1) and taking Fourier transforms give us

$$
I_{\epsilon}=\int \mathrm{e}^{i \varphi(q)} \widehat{b_{\epsilon, q}}\left(\xi, \eta,-\partial_{3} \varphi(q)\right) \widehat{u_{k}}\left(-\xi-\partial_{1} \varphi(q)\right) \widehat{v_{l}}\left(-\eta-\partial_{2} \varphi(q)\right) \frac{d \xi d \eta d q}{(2 \pi)^{2 n}} .
$$

This is the expression which will allow us not only to evaluate $\lim _{\epsilon \rightarrow 0} I_{\epsilon}$, but to get the $L^{2}$ estimate as well. To this end, by applying Taylor's formula, we write $b_{\epsilon, q}(z)=b_{q}(z) \psi(\epsilon m)+\epsilon r_{\epsilon, q}(z)$ where $b_{q}=b_{0, q}$ and

$$
r_{\epsilon, q}(z)=b_{q}(z) \sum_{j=1}^{\nu} \theta_{j} \int_{0}^{1} \partial_{j} \psi(\epsilon m+\epsilon t \theta) d t .
$$

To this corresponds the (a priori formal) decomposition : $I_{\epsilon}=I_{\epsilon}^{\prime}+\epsilon I_{\epsilon}^{\prime \prime}$ with

$$
I_{\epsilon}^{\prime}=\int \mathrm{e}^{i \varphi(q)} \widehat{b_{q}}\left(\xi, \eta,-\partial_{3} \varphi(q)\right) \psi(\epsilon m) \widehat{u_{k}}\left(-\xi-\partial_{1} \varphi(q)\right) \widehat{v_{l}}\left(-\eta-\partial_{2} \varphi(q)\right) \frac{d \xi d \eta d q}{(2 \pi)^{2 n}} .
$$

We shall prove that $I_{\epsilon}^{\prime}$ and $I_{\epsilon}^{\prime \prime}$ are well defined Lebesgue integrals and that we can pass to the limit in $I_{\epsilon}$. Consider $I_{\epsilon}^{\prime}$ and let us show that

$$
(\xi, \eta, q) \mapsto \widehat{b_{q}}\left(\xi, \eta,-\partial_{3} \varphi(q)\right) \widehat{u_{k}}\left(-\xi-\partial_{1} \varphi(q)\right) \widehat{v_{l}}\left(-\eta-\partial_{2} \varphi(q)\right)
$$

is an integrable function in $\mathbb{R}^{4 n+\nu}$. By Cauchy-Schwarz inequality, we have

$$
\begin{aligned}
J & \stackrel{\text { def }}{=} \int\left|\widehat{b_{q}}\left(\xi, \eta,-\partial_{3} \varphi(q)\right) \widehat{u_{k}}\left(-\xi-\partial_{1} \varphi(q)\right) \widehat{v_{l}}\left(-\eta-\partial_{2} \varphi(q)\right)\right| d \xi d \eta d q \\
& \leq\left(\int\left|\widehat{b_{q}}\right|\left|\widehat{u_{k}}\right|^{2} d \xi d \eta d q\right)^{\frac{1}{2}}\left(\int\left|\widehat{b_{q}}\right|\left|\widehat{v_{l}}\right|^{2} d \xi d \eta d q\right)^{\frac{1}{2}} \cdot
\end{aligned}
$$

Set $\beta=\sup _{q}\left|\widehat{b_{q}}\right|$. This is an integrable function in $\mathbb{R}^{2 n+\nu}$. Performing the changes of variables

$$
\left\{\begin{array} { l } 
{ l ^ { \prime } = \partial _ { 1 } \varphi ( k , l , m ) } \\
{ m ^ { \prime } = \partial _ { 3 } \varphi ( k , l , m ) }
\end{array} \quad \text { and } \quad \left\{\begin{array}{l}
k^{\prime}=\partial_{2} \varphi(k, l, m) \\
m^{\prime}=\partial_{3} \varphi(k, l, m)
\end{array}\right.\right.
$$

respectively in the first and second factors of the right hand side of the last inequality and using the assumption (2.3), we get

$$
\begin{aligned}
J^{2} \leq & \int \beta\left(\xi, \eta,-m^{\prime}\right)\left|\widehat{u_{k}}\left(-\xi-l^{\prime}\right)\right|^{2} \frac{d \xi d \eta d k d l^{\prime} d m^{\prime}}{\delta} \\
& \times \int \beta\left(\xi, \eta,-m^{\prime}\right)\left|\widehat{v}_{l}\left(-\eta-k^{\prime}\right)\right|^{2} \frac{d \xi d \eta d k^{\prime} d l d m^{\prime}}{\delta} \\
\leq & \frac{\operatorname{cst}}{\delta^{2}} \int \beta(\xi, \eta, m)\left|\widehat{u_{k}}(l)\right|^{2} d \xi d \eta d q \int \beta(\xi, \eta, m)\left|\widehat{v}_{l}(k)\right|^{2} d \xi d \eta d q \\
\leq & \frac{\operatorname{cst}}{\delta^{2}}\left(\int \beta(\zeta) d \zeta\right)^{2}|u|_{0}|v|_{0} .
\end{aligned}
$$


This and (3.4) imply that $J \leq \frac{\mathrm{cst}}{\delta}\|a\|_{S_{w}} \exp \left(\operatorname{cst}\left\|\varphi^{\prime \prime}\right\|_{S_{w}}\right)|u|_{0}|v|_{0}$. Hence, (3.5) is Lebesgue integrable in $\mathbb{R}^{4 n+\nu}$. Applying Lebesgue dominated convergence theorem yields the convergence of $I_{\epsilon}^{\prime}$ to

$$
I=\int \mathrm{e}^{i \varphi(q)} \widehat{b_{q}}\left(\xi, \eta,-\partial_{3} \varphi(q)\right) \widehat{u_{k}}\left(-\xi-\partial_{1} \varphi(q)\right) \widehat{v_{l}}\left(-\eta-\partial_{2} \varphi(q)\right) \frac{d \xi d \eta d q}{(2 \pi)^{2 n}} .
$$

The discussion of $I_{\epsilon}^{\prime \prime}$ is similar. Indeed, by an easy argument, one can show that

$$
\int \sup _{q}\left|\widehat{r_{\epsilon, q}}(\zeta)\right| d \zeta \leq \operatorname{cst}\left\|a_{\epsilon}\right\|_{S_{w}} \exp \left(\operatorname{cst}\left\|\varphi^{\prime \prime}\right\|_{S_{w}}\right) \leq \operatorname{cst}\|a\|_{S_{w}} \exp \left(\operatorname{cst}\left\|\varphi^{\prime \prime}\right\|_{S_{w}}\right)
$$

Consequently, arguing as for $I_{\epsilon}^{\prime}$ yields the fact that $I_{\epsilon}^{\prime \prime}$ is bounded with respect to $\epsilon$, so that $\lim _{\epsilon \rightarrow 0} I_{\epsilon}=I$ which shows that $A$ is an operator from $\mathcal{S}\left(\mathbb{R}^{n}\right)$ to $\mathcal{S}^{\prime}\left(\mathbb{R}^{n}\right)$ independent of $\psi$. Moreover, since

$$
|I| \leq J \leq \frac{\operatorname{cst}}{\delta}\|a\|_{S_{w}} \exp \left(\operatorname{cst}\left\|\varphi^{\prime \prime}\right\|_{S_{w}}\right)|u|_{0}|v|_{0}, \quad u, v \in \mathcal{S}\left(\mathbb{R}^{n}\right)
$$

$A$ extends uniquely to a bounded operator in $L^{2}\left(\mathbb{R}^{n}\right)$ whose norm satisfies the desired estimate. Theorem 3.1 is so proved.

Next, we give a last result on $L^{2}$ boundedness for Fourier integral operators related to the class $S_{w}$.

Theorem 3.3. Let a be in $H_{u l}^{s, s^{\prime}, \sigma}\left(\mathbb{R}^{n} \times \mathbb{R}^{n} \times \mathbb{R}^{\nu}\right), s, s^{\prime}>\frac{n}{2}, \sigma>\nu$, all the other assumptions being as in Theorem 3.1. Then, concerning the operator A, we have the same conclusion as that of Theorem 3.1, with, of course, $|a|_{s, s^{\prime}, \sigma ; u l}$ replacing $\|a\|_{S_{w}}$ in the estimate.

Proof. Since the proof is similar to that of Theorem 3.1, we only show how to get the basic estimate, that is, how to estimate the integral

$$
\left.I=\int \mathrm{e}^{i \varphi(q)} \widehat{b_{q}}\left(\xi, \eta,-\partial_{3} \varphi(q)\right) \widehat{u_{k}}\left(-\xi-\partial_{1} \varphi(q)\right){\widehat{v_{l}}}_{(-\eta}-\partial_{2} \varphi(q)\right) \frac{d \xi d \eta d q}{(2 \pi)^{2 n}}
$$

the notations being that of the proof of Theorem 3.1. Write $b_{q}=\chi a_{q} \mathrm{e}^{i \varphi_{q}}$ with some appropriate $\chi \in \mathcal{D}\left(\mathbb{R}^{2 n+\nu}\right)$ and set $\gamma(\zeta)=\sup _{q}\left|\mathcal{F}\left(\chi \mathrm{e}^{i \varphi_{q}}\right)(\zeta)\right|$. It follows easily from (3.3) that $\gamma$ is an integrable function in $\mathbb{R}^{2 n+\nu}$ and that $\int \gamma(\zeta) d \zeta \leq \operatorname{cst} \exp \left(\operatorname{cst}\left\|\varphi^{\prime \prime}\right\|_{S_{w}}\right)$. If $\zeta=\left(\zeta_{1}, \zeta_{2}, \zeta_{3}\right) \in \mathbb{R}^{n} \times \mathbb{R}^{n} \times \mathbb{R}^{\nu}$, we estimate 
as follows :

$$
\begin{aligned}
& |I| \leq \int \gamma(-\zeta) \times \\
& \left|\widehat{a_{q}}\left(\zeta_{1}-\xi, \zeta_{2}-\eta, \zeta_{3}-\partial_{3} \varphi(q)\right) \widehat{u_{k}}\left(\xi-\partial_{1} \varphi(q)\right) \widehat{v_{l}}\left(\eta-\partial_{2} \varphi(q)\right)\right| \frac{d \xi d \eta d q d \zeta}{(2 \pi)^{4 n+\nu}} \\
& \leq \int\left(\int\left\langle\xi-\zeta_{1}\right\rangle^{2 s}\left\langle\eta-\zeta_{2}\right\rangle^{2 s^{\prime}}\left\langle\zeta_{3}-\partial_{3} \varphi(q)\right\rangle^{2 \sigma}\right. \\
& \quad \times\left(\int \frac{\left.\left|\widehat{a_{q}}\left(\zeta_{1}-\xi, \zeta_{2}-\eta, \zeta_{3}-\partial_{3} \varphi(q)\right)\right|^{2} d \xi d \eta\right)^{\frac{1}{2}}}{\left\langle\xi-\zeta_{1}\right\rangle^{2 s}\left\langle\eta-\zeta_{2}\right\rangle^{2 s^{\prime}}\left\langle\zeta_{3}-\partial_{3} \varphi(q)\right\rangle^{2 \sigma}}\right)^{\frac{\gamma}{u_{l}}(-\zeta) d q d \zeta} \frac{(2 \pi)^{4 n+\nu}}{\left|\widehat{u_{k}}(\xi)\right|^{2} d \xi d q} \\
& \quad \leq \sup _{q}\left|\widehat{a_{q}}\right|_{s, s^{\prime}, \sigma} \int\left(\int \frac{\partial^{2}}{\left\langle\xi-\zeta_{1}+\partial_{1} \varphi(q)\right\rangle^{2 s}\left\langle\zeta_{3}-\partial_{3} \varphi(q)\right\rangle^{\sigma}}\right)^{\frac{1}{2}} \\
& \quad \times\left(\int \frac{\left|\widehat{v}_{l}(\eta)\right|^{2} d \eta d q}{\left\langle\eta-\zeta_{2}+\partial_{2} \varphi(q)\right\rangle^{2 s^{\prime}}\left\langle\zeta_{3}-\partial_{3} \varphi(q)\right\rangle^{\sigma}}\right)^{\frac{1}{2}} \frac{\gamma(-\zeta) d \zeta}{(2 \pi)^{4 n+\nu}}
\end{aligned}
$$

Here, we used essentially Cauchy-Schwarz inequality and the following elementary lemma whose proof is left to the reader (this is Lemma 1 of [5]):

Lemma 3.4. For all $s \in \mathbb{R}$, all compact sets $K \subset \mathbb{R}^{n}$ and all $\alpha \in \mathbb{N}^{n}$, there exists a constant $C_{s, \alpha, K}>0$ such that $\left|x^{\alpha}(1-\Delta)^{\frac{s}{2}} u(x)\right|_{0} \leq C_{s, \alpha, K}|u|_{s}$ for all $u \in H^{s}\left(\mathbb{R}^{n}\right)$ with support in $K$.

Performing the changes of variables (3.6) and estimating, we get :

$$
\begin{aligned}
&(2 \pi)^{4 n+\nu} \delta|I| \leq\left|a_{q}\right|_{s, s^{\prime}, \sigma ; u l} \int \gamma(\zeta)\left(\int \frac{\left|\widehat{u_{k}}(\xi)\right|^{2} d \xi d k d l^{\prime} d m^{\prime}}{\left\langle\xi+\zeta_{1}+l^{\prime}\right\rangle^{2 s}\left\langle m^{\prime}+\zeta_{3}\right\rangle^{\sigma}}\right)^{\frac{1}{2}} \\
& \times\left(\int \frac{\left|\widehat{v}_{l}(\eta)\right|^{2} d \eta d k^{\prime} d l d m^{\prime}}{\left\langle\eta+\zeta_{2}+k^{\prime}\right\rangle^{2 s^{\prime}}\left\langle m^{\prime}+\zeta_{3}\right\rangle^{\sigma}}\right)^{\frac{1}{2}} d \zeta \\
& \leq \operatorname{cst}\left|a_{q}\right|_{s, s^{\prime}, \sigma ; u l} \int \gamma(\zeta) d \zeta|u|_{0}|v|_{0} \\
& \leq \operatorname{cst}\left|a_{q}\right|_{s, s^{\prime}, \sigma ; u l} \exp \left(\operatorname{cst}\left\|\varphi^{\prime \prime}\right\|_{S_{w}}\right)|u|_{0}|v|_{0} .
\end{aligned}
$$

We do not go further into the proof.

Remark 3.5. This remark concerns the comparison between the spaces $S_{w}\left(\mathbb{R}^{2 n+\nu}\right)$ and $H_{u l}^{s, s^{\prime}, \sigma}\left(\mathbb{R}^{n} \times \mathbb{R}^{n} \times \mathbb{R}^{\nu}\right), s, s^{\prime}>\frac{n}{2}, \sigma>\nu$. This is important since it explains why we gave above three similar statements : Theorem 2.1, Theorem 3.1 and Theorem 3.3. We show here that both inclusions $S_{w}\left(\mathbb{R}^{2 n+\nu}\right) \subset H_{u l}^{s, s^{\prime}, \sigma}\left(\mathbb{R}^{n} \times \mathbb{R}^{n} \times \mathbb{R}^{\nu}\right)$ and $H_{u l}^{s, s^{\prime}, \sigma}\left(\mathbb{R}^{n} \times \mathbb{R}^{n} \times \mathbb{R}^{\nu}\right) \subset S_{w}\left(\mathbb{R}^{2 n+\nu}\right)$ are false in general. First, we have the following proposition : 
Proposition 3.6. The inclusion

$$
H_{u l}^{s_{1}, s_{2}, s_{3}}\left(\mathbb{R}^{n_{1}} \times \mathbb{R}^{n_{2}} \times \mathbb{R}^{n_{3}}\right) \subset S_{w}\left(\mathbb{R}^{n_{1}+n_{2}+n_{3}}\right)
$$

holds if $s_{i}>n_{i}, i=1,2,3$, and is false if $n_{1}=n_{2}=n_{3}=n$ and $s_{3}=n$.

Proof. If $u \in H_{u l}^{s_{1}, s_{2}, s_{3}}$ and $\chi, \tilde{\chi} \in \mathcal{D}, \tilde{\chi}=1$ on $\operatorname{supp}(\chi)$, by Cauchy-Schwarz inequality, we have

$$
\begin{aligned}
\left|\mathcal{F}\left(u \tau_{k} \chi\right)(\xi)\right| \leq(2 \pi)^{-n_{1}-n_{2}-n_{3}} & \left(\int\left|\left\langle\eta_{1}\right\rangle^{s_{1}}\left\langle\eta_{2}\right\rangle^{s_{2}}\left\langle\eta_{3}\right\rangle^{s_{3}} \mathcal{F}\left(u \tau_{k} \widetilde{\chi}\right)(\eta)\right|^{2} d \eta\right)^{\frac{1}{2}} \\
& \times\left(\int \frac{\left|\widehat{\tau_{k} \chi}(\xi-\eta)\right|^{2} d \eta}{\left\langle\eta_{1}\right\rangle^{2 s_{1}}\left\langle\eta_{2}\right\rangle^{2 s_{2}}\left\langle\eta_{3}\right\rangle^{2 s_{3}}}\right)^{\frac{1}{2}}
\end{aligned}
$$

where we used the notation $\eta=\left(\eta_{1}, \eta_{2}, \eta_{3}\right) \in \mathbb{R}^{n_{1}} \times \mathbb{R}^{n_{2}} \times \mathbb{R}^{n_{3}}$. Applying the inequalities $\left\langle\eta_{i}\right\rangle^{-2 s_{i}} \leq \operatorname{cst}\left\langle\xi_{i}\right\rangle^{-2 s_{i}}\left\langle\eta_{i}-\xi_{i}\right\rangle^{2 s_{i}}, i=1,2,3$, we obtain

$$
\left|\mathcal{F}\left(u \tau_{k} \chi\right)(\xi)\right| \leq \operatorname{cst} \frac{|u|_{s_{1}, s_{2}, s_{3} ; u l}}{\left\langle\xi_{1}\right\rangle^{s_{1}}\left\langle\xi_{2}\right\rangle^{s_{2}}\left\langle\xi_{3}\right\rangle^{s_{3}}}
$$

the constant being independent of $k$. Since the right hand side of this inequality is integrable, we have proved the first part of the proposition.

Consider now the function $a(x, y, \theta)=\langle x-y\rangle^{-n} \mathrm{e}^{-\theta^{2}-i(x-y) \theta}$. One can check (see also [8]) that the operator given by (2.1) is not bounded in $L^{2}\left(\mathbb{R}^{n}\right)$. It follows from Theorem 2.1 or Theorem 3.1 that $a \notin S_{w}\left(\mathbb{R}^{3 n}\right)$. Now, a direct computation shows that

$$
\partial_{x}^{\alpha} \partial_{y}^{\beta} \partial_{\theta}^{\gamma} a \in L^{\infty}\left(\mathbb{R}^{3 n}\right) \text { for all } \alpha, \beta, \gamma \in \mathbb{N}^{n},|\gamma| \leq n \text {. }
$$

Hence, $a \in H_{u l}^{s_{1}, s_{2}, n}\left(\mathbb{R}^{n} \times \mathbb{R}^{n} \times \mathbb{R}^{n}\right)$, for all $s_{1}, s_{2} \geq 0$, which proves the second part of the proposition.

Proposition 3.6 shows, after permutations of coordinates, that $H_{u l}^{s, s^{\prime}, \sigma}\left(\mathbb{R}^{n} \times \mathbb{R}^{n} \times \mathbb{R}^{\nu}\right)$, at least when $n=\nu$, is not contained in $S_{w}\left(\mathbb{R}^{2 n+\nu}\right)$ if $s \leq n$ or $s^{\prime} \leq n$ or $\sigma \leq \nu$. On the other hand, $S_{w}\left(\mathbb{R}^{2 n+\nu}\right)$ is neither contained in $H_{u l}^{s, s^{\prime}, \sigma}\left(\mathbb{R}^{n} \times \mathbb{R}^{n} \times \mathbb{R}^{\nu}\right)$, if $s, s^{\prime}>\frac{n}{2}, \sigma>\nu$, for $H_{u l}^{s, s^{\prime}, \sigma}\left(\mathbb{R}^{n} \times\right.$ $\left.\mathbb{R}^{n} \times \mathbb{R}^{\nu}\right) \subset C^{\tau}\left(\mathbb{R}^{2 n+\nu}\right)$ (Hölder space) if $0<\tau \leq \min \left\{s-\frac{n}{2}, s^{\prime}-\frac{n}{2}, \sigma-\frac{\nu}{2}\right\}$, as one can check easily, and the class $S_{w}$ contains functions that are merely continuous. Indeed, take $u(x)=\sum_{j \geq 1} \frac{1}{j^{2}} \mathrm{e}^{i 2^{j} x}$ in dimension 1. If $\chi \in \mathcal{S}(\mathbb{R})$, then, $\chi(D-k) u(x)=\sum_{j \geq 1} \frac{1}{j^{2}} \chi\left(2^{j}-k\right) \mathrm{e}^{i 2^{j} x}$, so that, $\int\|\chi(D-k) u\|_{L^{\infty}} d k \leq$ $\|\chi\|_{L^{1}} \sum_{j \geq 1} \frac{1}{j^{2}}<\infty$. Hence, $u \in S_{w}(\mathbb{R})$. However, $u$ is in no Hölder space $C^{\epsilon}(\mathbb{R}), \epsilon>0$, since it is given by a dyadic series.

As an application of the results above, we discuss now oscillating integrals with a large parameter in the phase. 
Corollary 3.7. Let $a$ and $\varphi$ be as in Theorem 2.1 or Theorem 3.1 or Theorem 3.3 , and let $A_{\lambda}, \lambda \geq 1$, be the operator defined from $\mathcal{S}$ to $\mathcal{S}^{\prime}$ by :

$$
A_{\lambda} v(x)=\int_{\mathbb{R}^{n+\nu}} \mathrm{e}^{i \lambda \varphi(x, y, \theta)} a(x, y, \theta) v(y) d y d \theta, \quad v \in \mathcal{S}\left(\mathbb{R}^{n}\right) .
$$

Then,

$$
\sup _{\lambda \geq 1} \lambda^{\frac{n+\nu}{2}}\left\|A_{\lambda}\right\|_{\mathcal{L}\left(L^{2}\right)} \leq \frac{\operatorname{cst}}{\delta} \exp \left(\operatorname{cst}\left\|\varphi^{\prime \prime}\right\|_{E}\right)\|a\|_{F}
$$

where $E$ (resp. $F)$ is one of the spaces $S_{w}\left(\mathbb{R}^{2 n+\nu}\right)$ and $H_{u l}^{s, s^{\prime}, \sigma}\left(\mathbb{R}^{n} \times \mathbb{R}^{n} \times\right.$ $\left.\mathbb{R}^{\nu}\right), s, s^{\prime}>\frac{n}{2}, \sigma>\nu$, and the constant is independent of $(a, \varphi, \delta)$.

Proof. We disregard the case $E=F=H_{u l}^{s, s^{\prime}, \sigma}$ since it is treated in [5]. Set $I(\lambda)=\left\langle A_{\lambda} v, u\right\rangle_{\mathcal{S}^{\prime}, \mathcal{S}}, u, v \in \mathcal{S}\left(\mathbb{R}^{n}\right)$ and write

$$
\lambda^{\frac{n+\nu}{2}} I(\lambda)=\lim _{\epsilon \rightarrow 0} \int_{\mathbb{R}^{2 n+\nu}} \mathrm{e}^{i \varphi_{\lambda}(x, y, \theta)} a_{\lambda}(x, y, \theta) \psi_{\lambda}(\epsilon \theta) u_{\lambda}(x) v_{\lambda}(y) d x d y d \theta
$$

with $u_{\lambda}(x)=\lambda^{-\frac{n}{4}} u\left(\lambda^{-\frac{1}{2}} x\right), v_{\lambda}(y)=\lambda^{-\frac{n}{4}} v\left(\lambda^{-\frac{1}{2}} y\right), \psi \in \mathcal{S}\left(\mathbb{R}^{\nu}\right), \psi(0)=1, \psi_{\lambda}(\theta)=$ $\psi\left(\lambda^{-\frac{1}{2}} \theta\right), a_{\lambda}(x, y, \theta)=a\left(\lambda^{-\frac{1}{2}}(x, y, \theta)\right), \varphi_{\lambda}(x, y, \theta)=\lambda \varphi\left(\lambda^{-\frac{1}{2}}(x, y, \theta)\right)$. Since $a_{\lambda}$ and $\varphi_{\lambda}$ satisfy the assumptions of Theorem 2.1 (resp. Theorem 3.1, Theorem 3.3), it follows from its conclusion that the integral (3.8) does not depend on $\psi$ and verifies the estimate :

$$
\left|\lambda^{\frac{n+\nu}{2}} I(\lambda)\right| \leq \frac{\mathrm{cst}}{\delta} \exp \left(\operatorname{cst}\left\|\varphi_{\lambda}^{\prime \prime}\right\|_{E}\right)\left\|a_{\lambda}\right\|_{F}|u|_{0}|v|_{0}, \quad \lambda \geq 1
$$

where $E=H_{u l}^{s, s^{\prime}, \sigma}, F=S_{w}$ (resp. $E=F=S_{w} ; E=S_{w}, F=H_{u l}^{s, s^{\prime}, \sigma}$ ). Now, applying Proposition 3.2 and its analogue concerning the $H_{u l}^{s, s^{\prime}, \sigma}$ space (see Proposition 1 of [5]), we obtain the corollary.

Notice that the estimate (3.7) is sufficiently explicit to allow one to treat the case where also the amplitude $a$ depends on the parameter $\lambda$, a case that often occurs in the applications and, for instance, in semi-classical pseudodifferential analysis.

We end this section by stating a particular (but important) case of the above results. This is the case where the phase and the amplitude do not depend on the $\theta$ variables (i.e. $\nu=0$ ). Unfortunately, this case is not a consequence of the preceding results except when only the space $S_{w}$ is involved. However, the proofs are similar to that above and even simpler since there is no problem to define the integrals. For this reason, we leave them to the reader.

Theorem 3.8. The estimate (3.7) also holds in the case $\nu=0$, that is, when $a$ and $\varphi$ do not depend on $\theta$ and the non-degeneracy condition (2.3) reduces to 
$\left|\operatorname{det} \partial_{x} \partial_{y} \varphi\right| \geq \delta$. In this case, $E$ (resp. F) stands for $S_{w}\left(\mathbb{R}^{2 n}\right)$ or $H_{u l}^{s, s^{\prime}}\left(\mathbb{R}^{n} \times\right.$ $\left.\mathbb{R}^{n}\right), s, s^{\prime}>\frac{n}{2}$, and $A_{\lambda}$ is given by

$$
A_{\lambda} v(x)=\int_{\mathbb{R}^{n}} \mathrm{e}^{i \lambda \varphi(x, y)} a(x, y) v(y) d y, \quad v \in \mathcal{S}\left(\mathbb{R}^{n}\right)
$$

As we said before, the case when $E=F=S_{w}\left(\mathbb{R}^{2 n}\right)$ is just a consequence of Corollary 3.7. Indeed, if $I(\lambda)=\left\langle A_{\lambda} v, u\right\rangle_{\mathcal{S}^{\prime}, \mathcal{S}}, u, v \in \mathcal{S}\left(\mathbb{R}^{n}\right)$, then,

$$
I(\lambda)=\lim _{\epsilon \rightarrow 0}\left(\frac{\lambda}{2 \pi}\right)^{n} \int_{\mathbb{R}^{3 n}} \mathrm{e}^{i \lambda \varphi_{1}(x, \eta, y)} a(x, y) \psi(\epsilon y) u(x) \widehat{v}(\lambda \eta) d x d y d \eta
$$

where $\psi \in \mathcal{S}\left(\mathbb{R}^{n}\right), \psi(0)=1$, and $\varphi_{1}(x, \eta, y)=\varphi(x, y)-y \eta$. Now, $\left|\operatorname{det} \widetilde{\varphi}_{1}^{\prime \prime}\right|=$ $\left|\operatorname{det} \partial_{x} \partial_{y} \varphi\right| \geq \delta$ and $(x, \eta, y) \mapsto a(x, y)$ is in $S_{w}\left(\mathbb{R}^{3 n}\right)$, so, applying Corollary 3.7 yields the desired result.

\section{On composition}

The spectral characterization of $S_{w}$ can also be used to discuss, in a natural manner, the composition of symbols in $S_{w}$.

For one thing, applying Theorem 1.2 or Corollary 1.3, one can reduce the problem, via decompositions, to composition of symbols in $S_{0,0}^{0}$. Since the composition in $S_{0,0}^{0}$ is already known and since it respects spectra, one can sum up the terms to obtain an element of $S_{w}$. We do not give the details of this since the idea is clear. For another thing, one can simplify Sjöstrand's proof ([1], [2]) based on the study of the action on $S_{w}$, by convolution, of non degenerate imaginary exponentials. Notice that this action is also useful to prove that the class of operators $\mathrm{Op}_{t}\left(S_{w}\right)$ does not depend on $t$. The spectral point of view allows us to give a simple proof of this action. In fact, the main (and simple!) idea is to study Fourier multipliers instead of convolutions. Notice that the formulas of the symbolic calculus, mainly in the non standard quantizations, are often written by means of Fourier multipliers.

Consider the Fourier multiplier $\mathrm{e}^{i \Phi(D)}$ where $\Phi$ is a quadratic form on $\mathbb{R}^{n}$ (which may be degenerate). We want to prove that this operator is bounded from $S_{w}$ to $S_{w}$. There is a symmetric matrix $M$ such that we have the classical (polarization) formula

$$
<M \xi, \eta>=\frac{\Phi(\xi+\eta)-\Phi(\xi)-\Phi(\eta)}{2}, \quad \xi, \eta \in \mathbb{R}^{n} .
$$

If $\chi \in \mathcal{S}\left(\mathbb{R}^{n}\right)$ and $u \in S_{w}\left(\mathbb{R}^{n}\right)$, by applying this formula, we can write

$$
\begin{aligned}
\chi(D-k) \mathrm{e}^{i \Phi(D)} u(x) & =\mathrm{e}^{i \Phi(k)-2 i<M k, k>} \mathrm{e}^{2 i<M D, k>+i \Phi(D-k)} \chi(D-k) u(x) \\
& =\mathrm{e}^{i \Phi(k)-2 i<M k, k>} \widetilde{\chi}(D-k) u(x+2 M k),
\end{aligned}
$$


where $\tilde{\chi}(\xi)=\mathrm{e}^{i \Phi(\xi)} \chi(\xi)$ is also in $\mathcal{S}\left(\mathbb{R}^{n}\right)$. Hence,

$$
\left\|\chi(D-k) \mathrm{e}^{i \Phi(D)} u\right\|_{L^{\infty}}=\|\tilde{\chi}(D-k) u\|_{L^{\infty}}
$$

so that

$$
\int_{\mathbb{R}^{n}}\left\|\chi(D-k) \mathrm{e}^{i \Phi(D)} u\right\|_{L^{\infty}} \leq \operatorname{cst}\|u\|_{S_{w}}
$$

which is the desired inequality.

Finally, we notice that one can even compose Fourier integral operators of type (2.2) satisfying $a \in S_{w}, \varphi^{\prime \prime} \in S_{w}$ and condition (2.3). Indeed, if $A_{1}, A_{2}$ are operators of the same type and are associated with amplitudes $a_{1}, a_{2}$ and phases $\varphi_{1}, \varphi_{2}$, then, $A_{1} A_{2}$ is given, at least formally, by the kernel

$$
\int \mathrm{e}^{i \varphi\left(x, z ; y, \theta, \theta^{\prime}\right)} a\left(x, z ; y, \theta, \theta^{\prime}\right) d y d \theta d \theta^{\prime}
$$

where $a\left(x, z ; y, \theta, \theta^{\prime}\right)=a_{1}(x, y, \theta) a_{2}\left(y, z, \theta^{\prime}\right)$ and $\varphi\left(x, z ; y, \theta, \theta^{\prime}\right)=\varphi_{1}(x, y, \theta)$ $+\varphi_{2}\left(y, z, \theta^{\prime}\right)$. The new " $\theta$-variables" are $\left(y, \theta, \theta^{\prime}\right)$. Clearly, $a, \varphi^{\prime \prime}$ are in $S_{w}\left(\mathbb{R}^{n} \times\right.$ $\left.\mathbb{R}^{n} \times \mathbb{R}^{n+\nu+\nu^{\prime}}\right)$ and the fact that the geometric condition (2.3) is satisfied by this $\varphi$ is easy to check and has been noticed by K. Asada and D. Fujiwara, [4]. Thus, the composite $A_{1} A_{2}$ is a Fourier integral operator of the same type.

\section{What about the action of changing variables?}

The one failing of $S_{w}$ is that it is not invariant under the action of a change of variables. Indeed, suppose that $h: \mathbb{R}^{n} \rightarrow \mathbb{R}^{n}$ is a diffeomorphism such that $h^{\star}(u)=u \circ h \in S_{w}\left(\mathbb{R}^{n}\right)$ for all $u \in S_{w}\left(\mathbb{R}^{n}\right)$. If $u$ has compact support the same holds for $h^{\star}(u)$. Using the definition of Sjöstrand, it is easy to check that $\left(S_{w}\right)_{\text {comp }}\left(\mathbb{R}^{n}\right)=\left(\mathcal{F} L^{1}\right)_{\text {comp }}\left(\mathbb{R}^{n}\right)$. Hence, $h^{\star}$ maps $\left(\mathcal{F} L^{1}\right)_{\text {comp }}\left(\mathbb{R}^{n}\right)$ into $\left(\mathcal{F} L^{1}\right)_{\text {comp }}\left(\mathbb{R}^{n}\right)$, and $\left(\mathcal{F} L^{1}\right)_{\text {loc }}\left(\mathbb{R}^{n}\right)$ into $\left(\mathcal{F} L^{1}\right)_{\text {loc }}\left(\mathbb{R}^{n}\right)$. It is then a classical fact that $h$ is necessarily an affine mapping, in other words, $h-h(0)$ is linear. We refer to Kahane [6] or Rudin [7]. Notice that in this argument, $h$ may be merely a continuous and proper mapping. Since an affine change of variables clearly preserves $S_{w}$, we have proved

Theorem 5.1. Let $h: \mathbb{R}^{n} \rightarrow \mathbb{R}^{n}$ be proper and continuous. If

$$
h^{\star}\left[S_{w}\left(\mathbb{R}^{n}\right)\right] \subset S_{w}\left(\mathbb{R}^{n}\right),
$$

then, $h$ is affine. The class $S_{w}$ is preserved by and only by affine changes of variables.

\section{Acknowledgements}

We thank N. Lerner for a stimulating discussion on this subject. We also thank J. Sjöstrand for his criticisms and suggestions which let us improve greatly the first version of this work. 


\section{References}

[1] J. Sjöstrand, An algebra of pseudodifferential operators, Math. Res. Lett. 1 (1994), 185192.

[2] W Wiener type algebras of pseudodifferential operators, Séminaire Equations aux Dérivées Partielles, Ecole Polytechnique, 1994/1995, Exposé ${ }^{\circ}$ IV.

[3] A. Boulkhemair, $L^{2}$ estimates for pseudodifferential operators, Ann. Scuola Norm. Sup. Pisa, Cl. Sci. (4) 22 (1995), 155-183.

[4] K. Asada and D. Fujiwara, On some oscillatory integral transformations in $L^{2}\left(\mathbb{R}^{n}\right)$, Japan. J. Math. 4 (1978).

[5] A. Boulkhemair, Estimations $L^{2}$ précisées pour des intégrales oscillantes, Prépublication de l'Université de Nantes, Rapport de Recherche 95/09-2.; Comm. Partial Differential Equations (to appear).

[6] J.-P. Kahane, Séries de Fourier absolument convergentes,, Ergeb. Math. Grenzgeb. (3), Springer-Verlag, Berlin, 1970.

[7] W. Rudin, Fourier analysis on groups, Intersci. Tracts Pure Appl. Math., 12, Interscience Publishers, New York-London, 1962.

[8] W. Rouhuai and L. Chengzhang, On the $L^{p}$ boundedness of several classes of pseudodifferential operators, Chin. Ann. Math. Ser. B 5 (1984), 193-213.

Université de Nantes, Département de Mathématiques, 2, Rue de la Houssinière, BP 92208, 44322 NANTES CEDEX 3, FRANCE

E-mail address: boulkhem@math.univ-nantes.fr 